\title{
How Generalizable Are Cardiovascular Outcome Trials of Sodium-Glucose Co-Transporter-2 Inhibitors? A National Database Study: Study Protocol
}

\author{
William Hinton $\cdot$ Michael D. Feher $\cdot$ Neil Munro $\cdot$ Simon de Lusignan (D
}

Received: March 13, 2019 / Published online: April 30, 2019

(c) The Author(s) 2019

\begin{abstract}
Introduction: Sodium-glucose co-transporter-2 (SGLT2) inhibitors are oral antihyperglycemic agents for the treatment of people with type 2 diabetes (T2DM). Two recent cardiovascular outcome trials (CVOTs), the EMPA-REG OUTCOME trial and CANVAS Program, have demonstrated that SGLT2 inhibitors have cardiovascular benefit in high-risk cardiovascular patients. The aim of our study will be to identify the prevalence of patients in an English primary care setting with the equivalent cardiovascular risk profile to those included in each of four SGLT2 inhibitor CVOTs: CANVAS, DECLARE, EMPA-REG, and VERTIS CV.
\end{abstract}

Enhanced Digital Features To view enhanced digital features for this article go to https://doi.org/10.6084/ m9.figshare.7982885.

Electronic Supplementary Material The online version of this article (https://doi.org/10.1007/s13300019-0620-8) contains supplementary material, which is available to authorized users.

W. Hinton · M. D. Feher · N. Munro ·

S. de Lusignan ( $\square)$

Department of Clinical and Experimental Medicine, University of Surrey, Guildford GU2 7XP, UK

e-mail: s.lusignan@surrey.ac.uk

S. de Lusignan

Royal College of General Practitioners (RCGP)

Research and Surveillance Centre (RSC), London, UK
Methods: Routinely collected primary care data from the Royal College of General Practitioners (RCGP) Research and Surveillance Centre (RSC) network database will be used. We will perform a cross-sectional analysis to calculate the prevalence of people that have equivalent cardiovascular risk to participants included in each of the four above-mentioned SGLT2 inhibitor CVOTs. The demographic and clinical characteristics of the subgroups will also be compared with participants in each trial. The study cohort will include people with T2DM in the RCGP RSC dataset. Subgroups of people will be identified using Read codes that most closely match the inclusion criteria of each trial. Descriptive statistics will be used to report the characteristics of people at high cardiovascular risk and compared against those of people in each CVOT.

Planned Outputs: Findings from the study will be submitted for publication in a peer-reviewed journal to report the applicability of each SGLT2 inhibitor trial to real-world clinical practice.

Funding: AstraZeneca UK Limited.

Keywords: Cardiovascular diseases; Crosssectional studies; Diabetes mellitus, Type 2; Medical record systems, computerized; Sodium-glucose transporter 2 inhibitors 


\section{INTRODUCTION}

Sodium-glucose co-transporter-2 (SGLT2) inhibitors belong to a relatively new class of oral antihyperglycemic agents for the treatment of people with type 2 diabetes (T2DM). They ameliorate glycemic control by preventing the reabsorption of glucose by SGLT2 in the proximal convoluted tubule of the kidney [1]. This process occurs independently of the actions of insulin and allows the drug to be used at any stage of diabetes progression while minimizing the risk of hypoglycemia [2, 3]. Clinical trials have demonstrated additional benefits of SGLT2 inhibitor therapy, including weight reduction and decreased blood pressure, both of which are thought to be due to increased excretion of glucose and sodium by the kidneys $[4,5]$. In additon, cardiovascular outcome trials (CVOTs) have been undertaken to demonstrate the safety in addition to efficacy of these antihyperglycemic agents.

In recent years two CVOTs have been conducted to explore whether SGLT2 inhibitors have cardioprotective effects in high-risk cardiovascular patients. In the Empagliflozin Cardiovascular Outcomes, and Mortality in Type 2 Diabetes Mellitus Patients-Removing Excess Glucose (EMPA-REG) outcome trial, time-toevent analysis confirmed that death due to cardiovascular causes, non-fatal myocardial infarction, or nonfatal stroke was less likely to occur in patients treated with empagliflozin than those given a placebo [6]. Similarly, the Canagliflozin Cardiovascular Assessment Study (CANVAS and CANVAS-R) Program showed that those treated with canagliflozin and followedup over a mean of 3.6 years were at a reduced risk of having a cardiovascular event than those assigned to the placebo group [7]. Both trials, therefore, confirmed non-inferiority with regard to cardiovascular safety of each SGLT2 inhibitor, as well as superiority in terms of primary outcome events.

Further trials have been carried out more recently to explore whether other SGLT2 inhibitors used to treat T2DM patients have similar cardiovascular benefits. The multicenter trial to evaluate the effect of Dapagliflozin on the
Incidence of Cardiovascular Events ([DECLARE])-TIMI 58; referred to hereafter as the DECLARE trial) demonstrated non-inferiority of dapagliflozin for major adverse cardiovascular events in patients treated with this drug compared to placebo, as well as significantly reduced hospitalization for heart failure or cardiovascular death [8]. Another CVOT, the Cardiovascular Outcomes Following Ertugliflozin Treatment in Type 2 Diabetes Mellitus Participants with Vascular Disease (VERTIS CV) trial, is estimated to be completed in September 2019 [9]. However, despite the cardiovascular safety/efficacy demonstrated by CVOTs, the real-world applicability of these findings to patients in clinical practice is uncertain.

We have previously compared the cardiovascular risk profile of T2DM patients in an English primary care dataset with that of trial participants using the inclusion criteria of the EMPA-REG trial [10]. Our findings showed that the results of the EMPA-REG trial were only applicable to a small proportion of people with $\mathrm{T} 2 \mathrm{DM}$, and to an even smaller proportion of those prescribed an SGLT2 inhibitor. The applicability of other SGLT2 inhibitor CVOTs to real-world clinical practice in a primary care setting is yet to be elucidated.

In this protocol we describe the method which will be used in our study to compare the cardiovascular risk profile of patients in a realworld primary care setting to that of participants in each of the four SGLT2 inhibitor CVOTs mentioned in the preceding text (CANVAS Program, DECLARE, EMPA-REG, and VERTIS CV). The results will inform the extent to which the previous and upcoming findings of each trial can be generalized to a real-world setting.

\section{OBJECTIVES}

The aim of the study is to identify all adult patients with T2DM in the Royal College of General Practitioners (RCGP) Research and Surveillance Center (RSC) database that meet the inclusion criteria of each of the four CVOTs for treatment with an SGLT2 inhibitor. An additional aim is to compare the demographic 
and clinical characteristics of identified patients with participants included in these trials.

\section{Primary Objectives}

1. To establish the number of people in the RCGP RSC population that meet the inclusion criteria of each CVOT for treatment with an SGLT2 inhibitor.

2. To describe the characteristics of people eligible for each trial according to:
(a) type of cardiovascular disease/risk factor;
(b) duration of their diabetes; and
(c) the number on concurrent oral antihy- perglycemic medications or prescribed insulin.

3. To describe the demographic (age, gender, ethnicity) and clinical characteristics (glycated hemoglobin [HbA1c], body mass index, blood pressure, and renal function) of people identified in each trial.

\section{Secondary Objectives}

To determine the number of people on an SGLT2 inhibitor that meet each of the inclusion criteria of each of the four CVOTs.

\section{METHODS}

\section{Study Design}

The study will be a cross-sectional analysis of all adults with T2DM included in the RCGP RSC database, with the aim to identify people with an equivalent cardiovascular risk profile to that of those persons included in each of the SGLT2 inhibitor CVOTs: CANVAS Program, DECLARE, EMPA-REG, and VERTIS CV. We will use an updated dataset from our previous comparison with the EMPA-REG trial.

\section{Data Source}

The RCGP RSC is a long established primary care sentinel network [11], comprising computerized medical records (CMRs) for over 200 primary care practices across England and a population of over 2,000,000 registered patients. This nationally representative network set up a weekly returns service in 1964 for the surveillance of respiratory infections, including influenza [12, 13], but has more recently widened its remit to include research into longterm conditions, such as diabetes.

As with UK primary care more generally, the RCGP RSC data is registration based, so that every patient is registered with only one practice at a time. All patients have a unique patient identifier, the National Health Service (NHS) number. This unique patient identifier enables the transition of a patient's medical record to another practice when he/she moves to a different location and patient data to be linked with other datasets, including secondary care datasets[14].

CMR data in UK primary care are captured using Read codes [15]. These codes are used to collate data for diagnoses, processes of care (such as care pathways, referrals, etc.), prescriptions, and results from laboratory-based data. Data quality in UK primary care is high, dating back as far as 2004 due to the introduction of a pay-for-performance scheme, i.e., the Quality and Outcomes Framework (QOF), which was implemented to encourage clinicians to achieve set targets for the management of chronic diseases [16].

We will analyze data extracted from primary care practices up to 31 December 2016, which will include all patients with a T2DM diagnosis and aged $>18$ years. From this sample we will identify and report the proportion of those persons with cardiovascular risk/diseases similar to those of persons in each of the four CVOTs (CANVAS Program, DECLARE, EMPA-REG, and VERTIS CV). Demographic and clinical characteristics for each identified sample will be reported and subsequently compared with those of the CVOTs that have previously been published. In addition, missing data for each variable will be provided.

To protect patient data the RCGP RSC data is pseudonymized by NHS number. This study was classified as an "Audit of current practice", so specific ethical approval was not required. 


\section{Data Analysis}

We will identify people with T2DM using a twostep process, which we have previously described elsewhere [17]. Firstly, people with diabetes are identified according to presence of diagnostic codes, two or more results for $\mathrm{HbA1c}$ or plasma glucose that confirm diabetes, and antihyperglycemic medications (not including metformin). People are then categorized by diabetes type (type 1 DM [T1DM], T2DM, undetermined) via a seven-step algorithm, which considers medications, diagnosis codes specific to diabetes type, and other clinical characteristics specific to T1DM or T2DM.

To calculate prevalence within the T2DM cohort, we will use the high cardiovascular risk inclusion criteria for each SGLT2 inhibitor CVOT (Table 1). To identify people by cardiovascular risk, we will use the closest matching diagnosis codes or other codes available to define diagnosis of each risk factor (Electronic Supplementary Material Appendix Tables A1-A9).

\section{Statistical Methods}

Descriptive statistics will be used to report the findings. We will calculate the proportions of patients eligible for each trial. To describe the characteristics of each cohort, we will use percentages to report categorical data, and means (with standard deviations) and medians (with interquartile ranges) will be used to describe continuous data. Differences between crude rates will be explored using 95\% confidence intervals.

\section{Compliance with Ethics Guidelines}

Consent will not be required for these data. We will not process data for people where opt-out codes are present; these account for just over $2 \%$ of the RCGP RSC population [18]. The data will be pseudonymized and encrypted before uploading to the Clinical Informatics Research Group secure server. Personal data will not be identifiable. This study is considered to be an "Audit of current practice" when tested against the Health Research Authority (HRA)/Medical
Research Council (MRC) "Is my study research" tool and therefore does not require specific ethical approval [19]. Approval for use of the data was acquired from the RCGP RSC Study Approval Committee.

\section{Strengths and Limitations}

As mentioned in the Data Source section, the large sample size of this representative dataset and the high-level data completeness of the data are particular strengths of the RCGP RSC dataset. Furthermore, our previous study comparing real-world use of empagliflozin with data from a trial demonstrated that this type of study is feasible using the RCGP RSC dataset [10]. However, primary care data are associated with some limitations.

Practices participate in the RCGP RSC network on a voluntary basis, and there is slight underrepresentation of practices with more deprived patients compared to the national population [12]. Therefore, the sample is subject to some selection bias. In addition, the data collected are dependent on data entry into a patient's medical record, so data for particular conditions could be missing from some patients' records. Nonetheless, improved management of chronic diseases since the introduction of QOF will have minimized such an effect for this particular study on people with cardiovascular risk factors and T2DM [16].

Identification of patients according to trial inclusion criteria will also be restricted by primary care clinical codes, i.e., Read codes, which do not align directly with those used in the trials. Although we will use codes that most closely match those in the trials, this may lead to over- or underestimation of the number of people meeting each of the criteria. We will report additional strengths and limitations identified while undertaking the study in the final manuscript.

\section{CONCLUSIONS}

Our real-world evidence-based cross-sectional analysis will report the proportion of people with T2DM in a national primary care 


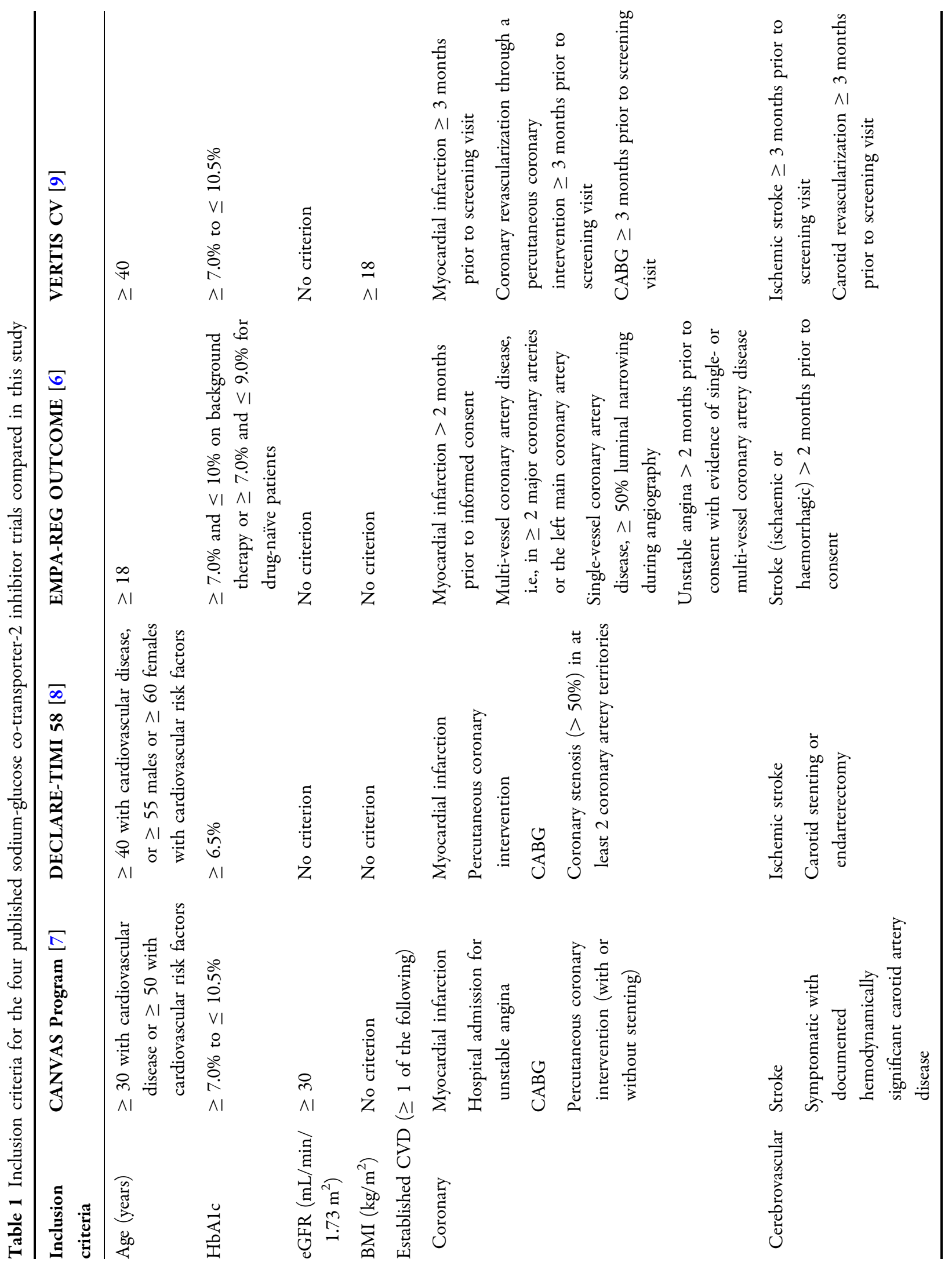




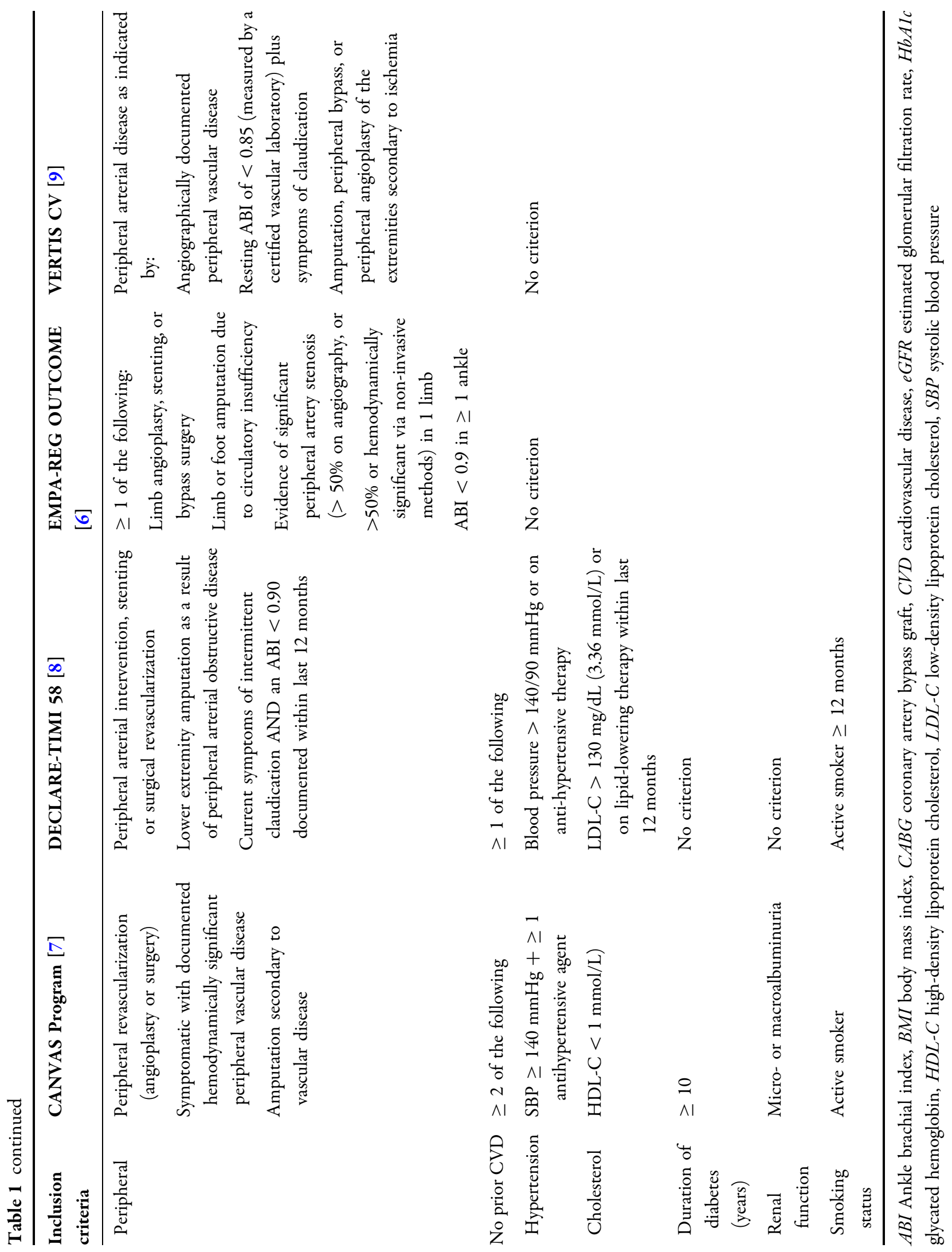


population that meet the cardiovascular risk inclusion criteria of each of the four drugspecific SGLT2 inhibitor CVOTs, with the aim to determine those deemed suitable for treatment as per each trial. The clinical characteristics of the identified patients in the RCGP RSC dataset will also be reported and compared with published findings from trials to determine their generalizability to real-world clinical practice.

\section{ACKNOWLEDGEMENTS}

The authors would like to thank patients and practices who are members of the RCGP RSC network, and the CMR system vendors: EMIS, In Practice, and TPP. We would also like to thank Apollo Medical systems, the RCGP, and University of Surrey colleague Filipa Ferreira (Senior Project Manager).

Funding. Sponsorship for this study and article processing charges were funded by AstraZeneca UK Limited (QC6114). All authors will have full access to all of the data in this study and will take complete responsibility for the integrity of the data and accuracy of the data analysis.

Authorship. All named authors meet the International Committee of Medical Journal Editors (ICMJE) criteria for authorship for this manuscript, take responsibility for the integrity of the work as a whole, and have given final approval for the version to be published.

Authorship Contributions. William Hinton led the drafting of the manuscript. Simon de Lusignan, Michael Feher, and Neil Munro reviewed the manuscript and study design, and contributed to the final manuscript draft.

Disclosures. William Hinton receives research funding from Eli Lilly and Company, Novo Nordisk Limited, and AstraZeneca UK Ltd. Simon de Lusignan receives research funding from Eli Lilly Company, GlaxoSmithKline, Takeda, AstraZeneca, and Novo Nordisk Limited. Neil Munro has received financial support for research, speaker meetings and consultancy from MSD, Merck, BMS, AstraZeneca, Pfizer, Novo Nordisk Limited, Eli Lilly and Company, and Sanofi-Aventis. Michael Feher has received financial support for research, speaker meetings, and consultancy from MSD, Merck, BMS, AstraZeneca, Pfizer, Novo Nordisk Limited, Eli Lilly and Company, and Sanofi-Aventis.

Compliance with Ethics Guidelines. To protect patient data the RCGP RSC data is pseudonymised by NHS number. This study was classed as an "Audit of current practice", so specific ethical approval was not required.

Data Availability. Data sharing is not applicable to this article as no datasets are to be generated or analyzed during the current study.

Open Access. This article is distributed under the terms of the Creative Commons Attribution-NonCommercial 4.0 International License (http://creativecommons.org/licenses/ by-nc/4.0/), which permits any noncommercial use, distribution, and reproduction in any medium, provided you give appropriate credit to the original author(s) and the source, provide a link to the Creative Commons license, and indicate if changes were made.

\section{REFERENCES}

1. Kalra S. Sodium glucose co-transporter-2 (SGLT2) inhibitors: a review of their basic and clinical pharmacology. Diabetes Ther. 2014;5(2):355-66.

2. Seufert J. SGLT2 inhibitors-an insulin-independent therapeutic approach for treatment of type 2 diabetes: focus on canagliflozin. Diabetes Metab Syndr Obes. 2015;8:543-54.

3. Wilding JP, Woo V, Rohwedder K, Sugg J, Parikh S, Dapagliflozin 006 Study Group. Dapagliflozin in patients with type 2 diabetes receiving high doses of insulin: efficacy and safety over 2 years. Diabetes Obes Metab. 2014;16(2):124-36.

4. Bolinder J, Ljunggren Ö, Kullberg J, et al. Effects of dapagliflozin on body weight, total fat mass, and regional adipose tissue distribution in patients with type 2 diabetes mellitus with inadequate glycemic 
control on metformin. J Clin Endocrinol Metab. 2012;97(3):1020-31.

5. Imprialos K, Stavropoulos K, Stavropoulos N, et al. Sodium-glucose cotransporter 2 inhibitors: impact on body weight and blood pressure compared with other antidiabetic drugs. Cardiovasc Hematol Disord Drug Targets. 2018;18:104-13.

6. Zinman B, Wanner C, Lachin JM, et al. Empagliflozin, cardiovascular outcomes, and mortality in type 2 diabetes. N Engl J Med. 2015;373(22):2117-28.

7. Neal B, Perkovic V, Matthews DR, et al. Canagliflozin and cardiovascular and renal events in type 2 diabetes. N Engl J Med. 2017;377(7):644-57.

8. Wiviott SD, Raz I, Bonaca MP, et al. Dapagliflozin and cardiovascular outcomes in type 2 diabetes. N Engl J Med. 2019;380(4):347-57.

9. Merck Sharp \& Dohme Corp. Cardiovascular outcomes following Ertugliflozin treatment in type 2 diabetes mellitus participants with vascular disease, The VERTIS CV Study (MK-8835-004). 2013. https://clinicaltrials.gov/ct2/show/NCT01986881. Accessed 4 Jul 2018.

10. McGovern A, Feher M, Munro N, de Lusignan S. Sodium-glucose co-transporter 2 (SGLT2) inhibitor: comparing trial data and real world use. Diabetes Ther. 2017;8(2):365-76.

11. Fleming DM. Weekly returns service of the Royal College of General Practitioners. Commun Dis Public Health. 1999;2(2):96-100.

12. Correa A, Hinton W, McGovern A, et al. Royal College of General Practitioners Research and
Surveillance Centre (RCGP RSC) sentinel network: a cohort profile. BMJ Open. 2016;6(4):e011092.

13. Elliot AJ, Fleming DM. Surveillance of influenza-like illness in England and Wales during 1966-2006. Euro Surveill. 2006;11(10):249-50.

14. de Lusignan S, van Weel C. The use of routinely collected computer data for research in primary care: opportunities and challenges. Fam Pract. 2006;23(2):253-63.

15. de Lusignan S. Codes, classifications, terminologies and nomenclatures: definition, development and application in practice. Inform Prim Care. 2005;13(1):65-70.

16. Langdown C, Peckham S. The use of financial incentives to help improve health outcomes: is the quality and outcomes framework fit for purpose? A systematic review. J Public Health (Oxf). 2014;36(2):251-8.

17. McGovern A, Hinton W, Correa A, Munro $\mathrm{N}$, Whyte $M$, de Lusignan S. Real-world evidence studies into 458 treatment adherence, thresholds for intervention and disparities in treatment in people with type 2 diabetes in the UK. BMJ Open. 2016;6(11):e012801.

18. Chan T, Di Iorio CT, de Lusignan S, Russo DL, Kuziemsky C, Liaw ST. UK National Data Guardian for Health and Care's Review of Data Security: trust, better security and opt-outs. J Innov Health Inform. 2016;23(3):627-32.

19. Health Research Authority. Is my study research? http://www.hra-decisiontools.org.uk/research/ (2017). Accessed 17 Jan 2018. 